\title{
Spiral imaging with off-resonance reconstruction for MRI-guided cardiovascular catheterizations using commercial off-the-shelf nitinol guidewires
}

\author{
Adrienne E Campbell-Washburn ${ }^{*}$, Toby Rogers ${ }^{1}$, Kanishka Ratnayaka ${ }^{1,2}$, Burcu Basar $^{1}$, Ozgur Kocaturk ${ }^{1,3}$, Hui Xue , \\ Robert J Lederman ${ }^{1}$, Michael S Hansen ${ }^{1}$, Anthony Z Faranesh ${ }^{1}$
}

From 19th Annual SCMR Scientific Sessions

Los Angeles, CA, USA. 27-30 January 2016

\section{Background}

MRI-guidance of cardiovascular catheterization offers improved soft-tissue contrast and reduced ionizing radiation exposure. The application of MRI-guidance to complex catheterization procedures has been limited by the unavailability of guidewires that are safe and visible under MRI. Here, we use RF-efficient spiral imaging for MR-guided cardiovascular catheterization, with real-time off-resonance reconstruction for improved visualization of off-the-shelf nitinol guidewires.

\section{Methods}

MRI-guided left and right heart catheterizations were performed on a swine using a commercial nitinol guidewire $(0.035 " / 145 \mathrm{~cm}$ Nitrex, Covidien, Plymouth, MN) and balloon-tipped catheter (7 Fr, Arrow-Teleflex, Limerick, PA) with spiral imaging (gradient echo, 16 interleaves, $\mathrm{TE} / \mathrm{TR}=0.86 / 11 \mathrm{~ms}$, flip $=10^{\circ}$, FOV $=$ $300 \mathrm{~mm} \times 300 \mathrm{~mm}$, matrix $=192 \times 192$, slice thickness $=6 \mathrm{~mm}$ ). To enhance guidewire visualization, we exploited the off-resonance signal near the guidewire. Using a custom reconstruction framework (Gadgetron [1]), the imaging data was reconstructed at two different off-resonance frequencies $( \pm 100 \mathrm{~Hz})$ and the images were subtracted to produce guidewire-enhanced images. The method was implemented such that operators could rapidly toggle between anatomical imaging, saturation pre-pulses for visualization of gadoliniumfilled balloon [2] and guidewire-enhanced imaging, as-needed throughout the procedure.

\footnotetext{
${ }^{1}$ Cardiovascular and Pulmonary Branch, Division of Intramural Research, National Heart, Lung, and Blood Institute, National Institutes of Health, Bethesda, MD, USA

Full list of author information is available at the end of the article
}

RF-induced heating of the guidewire/catheter configuration was evaluated in an ASTM 2182 phantom. A fiber-optic temperature probe $(0.007$ " OpSens, Quebec, Canada) affixed to the guidewire tip measured temperature during 2 minutes of continuous scanning with the spiral sequence and our standard real-time imaging sequence (Cartesian bSSFP, TE/TR $=1.31 / 2.62 \mathrm{~ms}$, flip angle $=45^{\circ}$.

\section{Results}

The spiral sequence generated 6 frames/s. Guidewireenhanced images offered improved delineation of the guidewire shaft, compared to standard signal void visualization (Figure 1A), and a unique guidewire tip artifact when in-plane (Figure 1B). These images also preserve tissue boundaries, which is valuable to provide anatomical context for guidewire navigation.

Substantial heating $\left(\Delta \mathrm{T}=80.5^{\circ} \mathrm{C}\right)$ was observed using our standard real-time Cartesian bSSFP sequence. Heating was reduced to below allowable limits using spiral gradient echo imaging $\left(\Delta \mathrm{T}=1.63^{\circ} \mathrm{C}\right)$ (Figure 2).

\section{Conclusions}

This visualization method is particularly flexible because it uses a targeted reconstruction of standard anatomical imaging data. This method may enable safe MRI-guided cardiovascular catheterizations using commercially available nitinol guidewires.

\footnotetext{
Authors' details

${ }^{1}$ Cardiovascular and Pulmonary Branch, Division of Intramural Research, National Heart, Lung, and Blood Institute, National Institutes of Health, Bethesda, MD, USA. Department of Cardiology, Children's National Medical Center, Washington, DC, USA. Institute of Biomedical Engineering, Bogazici University, Istanbul, Turkey, USA.
} 

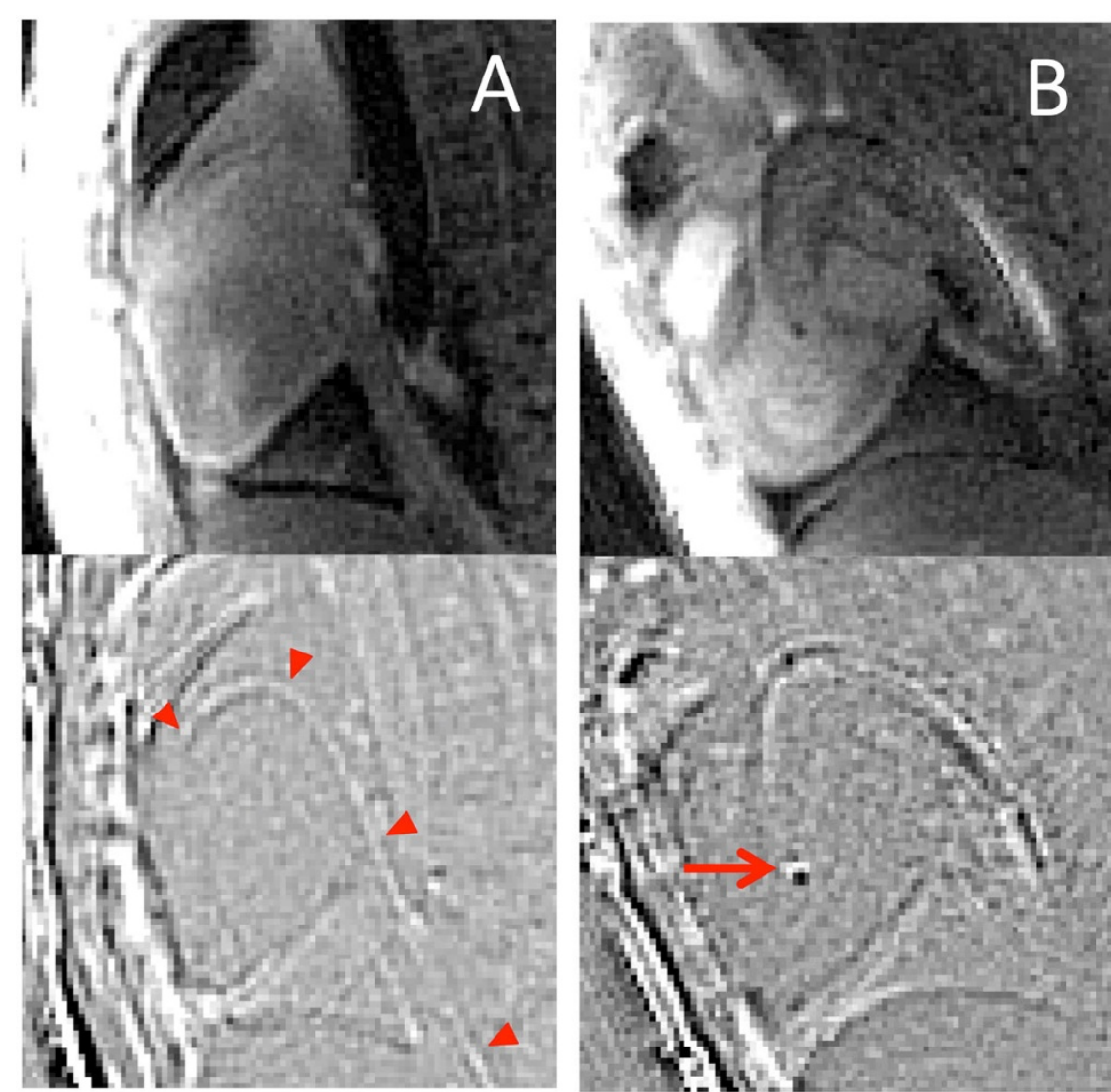

Figure 1 Images from right heart (A) and left heart (B) catheterizations, comparing standard anatomical imaging (top) to guidewireenhanced images (bottom). Improved guidewire visualization compared to signal-void imaging ( $A$, red arrowheads) and a unique in-plane guidewire tip signal ( $B$, red arrow) are demonstrated. Tissue boundaries are also visible in the guidewire-enhanced images which provides anatomical context for navigation.

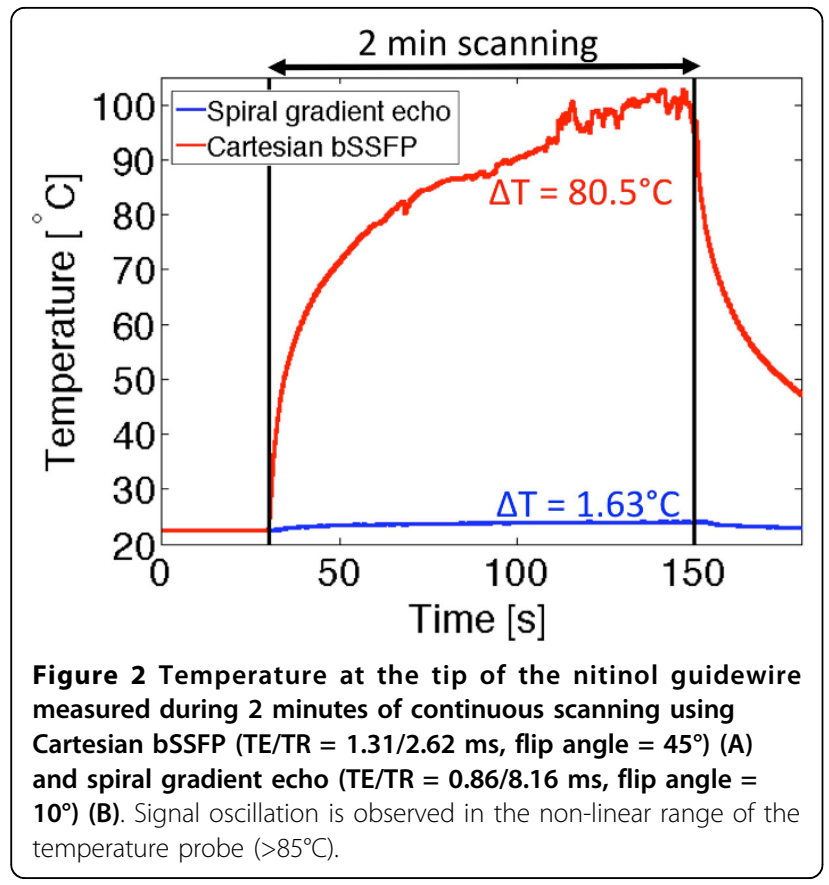

Published: 27 January 2016

\section{References}

1. Hansen MS, Sørensen TS: Gadgetron: an open source framework for medical image reconstruction. Magn Reson Med 2013, 69(6):1768-1776.

2. Ratnayaka K, Faranesh AZ, Hansen MS, Stine AM, Halabi M, Barbash IM, Schenke WH, Wright VJ, Grant LP, Kellman P, Kocaturk O, Lederman RJ: Real-time MRI-guided right heart catheterization in adults using passive catheters. Eur Heart J 2013, 34(5):380-389.

doi:10.1186/1532-429X-18-S1-P216

Cite this article as: Campbell-Washburn et al: Spiral imaging with offresonance reconstruction for MRI-guided cardiovascular catheterizations using commercial off-the-shelf nitinol guidewires. Journal of Cardiovascular Magnetic Resonance 2016 18(Suppl 1):P216. 